\title{
Modeling of Rotary Kilns and Application to Limestone Calcination
}

\author{
Uwe Küssel $^{1} \quad$ Dirk Abel $^{1} \quad$ Matthias Schumacher $^{2} \quad$ Martin Weng $^{2}$ \\ ${ }^{1}$ RWTH Aachen University, Institute of Automatic Control \\ Steinbachstraße 54A, D-52074 Aachen \\ ${ }^{2}$ Aixprocess, Process and Fluid Engineering \\ Alfonsstrasse 44, D-52070 Aachen
}

\begin{abstract}
This paper presents the one dimensional modeling of rotary kilns used for energy intensive production processes. Raw material is fed into an inclined rotating kiln and heated by counter current gas flow. Chemical reactions take place in the bed of raw material as well as in the gas phase. Heat and mass transfer between the bed and the gas phase are implemented. Also the heat transfer to the environment is taken into account. As a benchmark, the process of limestone calcination is chosen. Results are compared with computational fluid dynamic simulations.
\end{abstract}

Keywords: $\mathrm{CaCO}_{3}$, calcination, $\mathrm{CFD}$ comparison, kilns, limestone, rotary kilns, simulation

\section{Introduction}

Unhydrated lime is used as a raw material for many products in chemical industry. The limestone calcination, as an energy intensive production process for unhydrated lime, is often performed in continuously operating rotary kilns. Until today, the process is manually operated and despite existing approaches the use of automatic control is very uncommon. Due to the hot and dusty atmosphere inside the drum, thermodynamic states used as controlled variables by an expert system are hardly measurable in a reliable way. In heat driven chemical production processes such as limestone production measured data like a temperature as one thermodynamic variable of the process (often most easily to measure) is not sufficient to estimate the chemical rate of degradation of $\mathrm{CaCO}_{3}$. To overcome this problem dynamic physical and chemical models can be applied to predict the behavior of dependent measures. By comparing the results of the modeling to current plant measurements the states of independent variables become available to close the control loop. To be applicable to an expert system, the very complex model of the process has to be capable of real-time operation anyway and thus, several assumptions and simplifications have to be done.

As a first step to the automatic control of continuously operating rotary kilns, a detailed model of this process is developed. The model abstracts from reality by assuming one dimensional (1-D) counter current flow of the raw material phase (bed phase) and the gas phase. The bed and gas phase are surrounded by a combination of isolating refractory and steel shells. Two reactions are implemented, one for the production of heat and one for the calcination itself. Methane is oxidized in the gas phase in order to supply the necessary energy to the process and limestone is calcinated in the bed phase consuming energy due to an endothermic degradation process. Heat and mass transfer take place between the counter current flows and the environment. The paper is structured as follows: In chapter 2 the abstraction to 1-D counter current flow is shown. Additionally, the mechanisms of reactions and heat transfer are described. In chapter 3 the computational results with Dymola are analyzed in detail. For validation, the results are compared to computational fluid dynamics (CFD) simulations in chapter 4 . Chapter 5 concludes the paper with an outlook of future investigation.

\section{Modeling of rotary kilns}

The rotary kiln is modeled using a 1-D approach. The flows of gas and bed phase are counter current. The rotary kiln itself consists of an isolation and a steel shell. This abstraction is depicted in Figure 1. 


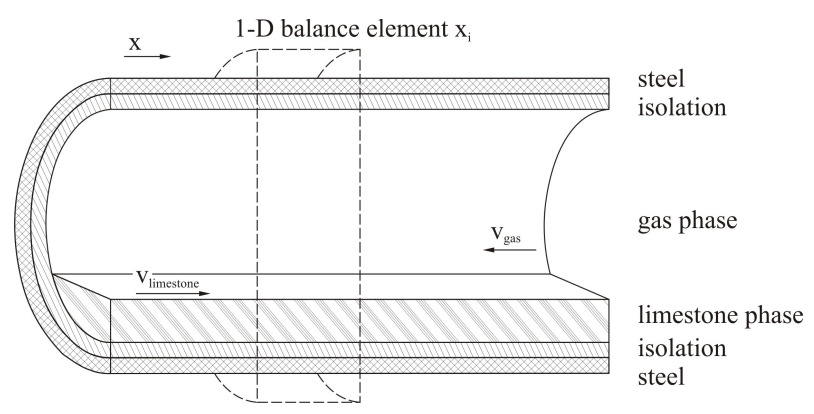

Figure 1: Rotary kiln with 1-D balance element

\subsection{Modeling of the gas phase}

The gas phase model is based on the DynamicPipe model of the Modelica fluid library [1]. It is extended by dynamically changing cross sectional areas due to bed heights. Replaceable models of radiative and convectional heat transfer are integrated and will be discussed in section 2.6. In addition, the gas phase is equipped with a replaceable chemical reaction model in order to cover the oxidation of mixed gaseous hydrocarbons (i.e. in this case Methane only), details are covered in section 2.5 .

The balancing equations with a dynamic momentum balance are formulated in [2]. Using a finite volume approximation and setting the momentum balance to be static, the equations (1)-(3), corresponding to a single slice used in the DynamicPipe model, are derived. To avoid obscurity, additional equations covering multi-component media are not given in this formulation.

$$
\begin{aligned}
\frac{d m_{i}}{d t}= & \dot{m}_{i+\frac{1}{2}}+\dot{m}_{i-\frac{1}{2}}+\cdots \\
0= & \frac{\dot{m}_{i+\frac{1}{2}}^{2}}{A_{i+\frac{1}{2}} p_{i+\frac{1}{2}}}-\frac{\dot{m}_{i-\frac{1}{2}}^{2}}{A_{i-\frac{1}{2}} p_{i-\frac{1}{2}}} \\
& +A_{i}\left(p_{i+\frac{1}{2}}-p_{i-\frac{1}{2}}\right) \\
& -F_{F_{F V}}-A_{i} \rho_{i} g \delta z \\
\frac{d U_{i}}{d t=} & \dot{H}_{i+\frac{1}{2}}+\dot{H}_{i-\frac{1}{2}} \\
& +v_{i} A_{i}\left(p_{i-\frac{1}{2}}-p_{i+\frac{1}{2}}\right)+\cdots \\
& \text { for slice } i=1, \ldots, n
\end{aligned}
$$

Source terms for heat, namely radiational and convectional contributions, are added to the right hand side (RHS) of the energy balance in equation (3). Mass transfer between bed and gas phase due to degradation of $\mathrm{CaCO}_{3}$ (releases $\mathrm{CO}_{2}$ ) and changes in the composition of the gas phase due to the oxidation of gaseous hydrocarbons are added to the RHS of the mass balance in equation (1). Including the heat of formation of all components implicitly adds the contribution of chemical reactions to the energy balance while only considering mass balance changes. For the longitudinal gas flow and the heat transfer standard connectors are used. For the gaseous exchange between gas and bed signal oriented connectors for each direction are designed using the same exchange medium $\left(\mathrm{O}_{2}, \mathrm{CO}\right.$, $\mathrm{CO}_{2}, \mathrm{H}_{2} \mathrm{O}$ ).

All cross sectional areas, and hence corresponding volumes, in these equations are dynamically changed due to changes in bed height. Therefore, the information of the bed's cross sectional area is transmitted via input output relation to the gas phase. Finally, data on gas phase properties are provided for other components by an output connector.

\subsection{Modeling of the bed phase}

The bed phase is modeled by mass and energy balances.

$$
\begin{array}{rll}
\frac{d m_{i}}{d t}= & \dot{m}_{i+\frac{1}{2}}+\dot{m}_{i-\frac{1}{2}}+\cdots \\
& \text { with } \quad \dot{m}_{i+\frac{1}{2}}=\rho_{i} \dot{V}_{i+\frac{1}{2}} \\
\frac{d U_{i}}{d t}= & \dot{H}_{i+\frac{1}{2}}+\dot{H}_{i-\frac{1}{2}}+\cdots
\end{array}
$$

Heat and mass transfer is included similarly to gas phase modelling via source terms to the RHS of the balance equations. The transport of material through the rotary kiln is specified by equation (6), which is commonly known as Kramers equation [5].

$$
\dot{V}_{i+\frac{1}{2}}=\frac{4}{3} \pi \omega R^{3}\left(\frac{\tan \alpha}{\sin \beta}-\frac{d h_{i}}{d x} \cot \beta\right)\left(2 \frac{h_{i}}{R}-\frac{h_{i}^{2}}{R^{2}}\right)^{\frac{3}{2}}
$$

The volume flow rate is a function of the rotation frequency $\omega$, the inner radius of the pipe $R$, the inclination of the kiln $\alpha$, the materials angle of repose $\beta$, the height of material $h$ and the corresponding gradient $\frac{d h}{d x}$. This differential equation in terms of the height is simplified by rearranging and approximating the height and the corresponding gradient with the equations (7) to (9).

$$
\begin{aligned}
h_{i} & =R-\left(R \cos \left(\varphi_{i} / 2\right)\right) \\
\frac{d h_{i}}{d x} & =(R / 2) \sin \left(\varphi_{i} / 2\right) \frac{d \varphi_{i}}{d x} \\
\frac{d \varphi_{i}}{d x} & \approx\left(\varphi_{i+1}-\varphi_{i}\right) /(L / n)
\end{aligned}
$$


The mid point angle of the bed phase is denoted by $\varphi$, the length of the rotary kiln by $L$ and $n$ is the number of slices in the rotary kiln. All geometric details for gas and bed phase are calculated using the mid point angle. The calculation of $\varphi_{i}=f\left(A_{i}\right)$ is presented in section 2.4.

\subsection{Modeling of the shells}

The isolation and steel shells are modeled by an energy balance for the corresponding material volume resulting from the 1-D balancing elements. Heat transfer, and hence the temperature profile, is realized using Fourier's relation for heat conduction in solid material. In principle, the shells are structured as grids consisting of heat capacitors and thermal conductors likewise modeled in the modelica standard package. Heat transfer is possible in two directions, namely longitudinally and transversely to the flow direction in the rotary kiln. Geometric and material properties are parameters of the model. Standard heat connectors are used.

\subsection{Mid point angle approximation}

All geometric properties for gas and bed phase can easily be calculated using the mid point angle $\varphi$ of the bed phase. Nevertheless, the angle has to be calculated from the cross sectional area of the bed phase, which is a direct result of the mass balance in every bed slice of the rotary kiln. This is shown in equation (10).

$$
A_{i}=\left(m_{i} / \rho_{i}\right) /(L / n)
$$

The relation between the cross sectional area $A_{i}$ of a single slice and the mid point angle $\varphi_{i}$ is implicitly given with the equations (11)-(12).

$$
\begin{aligned}
& 0=-P+\left(\varphi_{i}-\sin \varphi_{i}\right) \\
& P=2 A_{i} / R^{2}
\end{aligned}
$$

In order to avoid additional nonlinear equations, the relationship given with equation (11) is numerically solved for $\varphi$ in a range of $P$ between $1.25 \cdot 10^{-5}$ and $2 \pi$. The corresponding pairs are then used for a least square fit in order to find a good approximation for $\varphi=f(P)$. The function $\varphi=f(P)$ is given with equation (13).

$$
\begin{aligned}
\varphi= & a_{0} \log (P)+a_{1}(1 / P)+a_{2} P^{\frac{1}{2}} \\
& +a_{3} P+a_{4} P^{2}+a_{5} P^{3}+a_{6} P^{4} \\
& +a_{7} P^{5}+a_{8} P^{6}+a_{9}
\end{aligned}
$$

The error between the approximation and original data pairs is given in Figure 2.

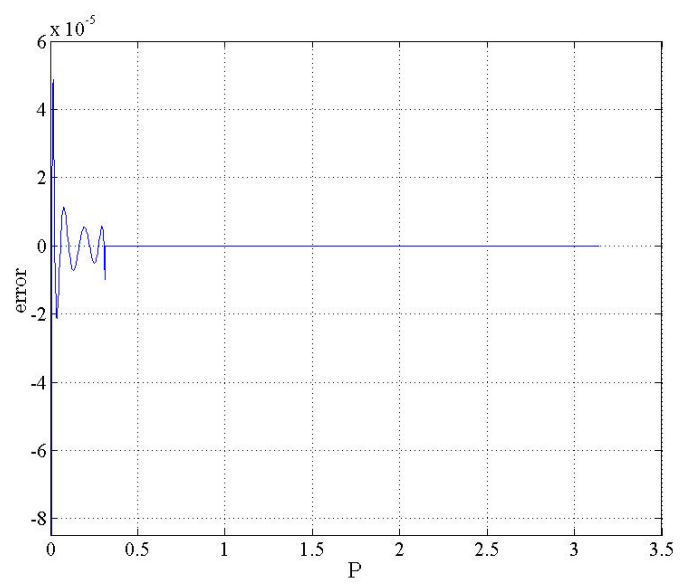

Figure 2: Approximation error for $\varphi=f(P)$

The adapted relationship $\varphi=f(P)$ is implemented as a function and integrated in both the gas and bed phase in order to calculate the mid point angle and subsequently all geometric characteristics of the rotary kiln.

\subsection{Mechanism of chemical reactions}

The replaceable chemical reaction model is designed as a reactant limited elementary reaction using an Arrhenius type approach for the reaction kinetics. The reaction kinetics approach is shown with the equation (14).

$$
k=B \cdot T^{n} \cdot e^{-\frac{E_{a}}{R \cdot T}}
$$

The preexponential coefficient $B$, the activation energy $E_{a}$ and $n$ are free parameters which will be used to fit the model to CFD simulation data. Generally speaking, this fit is necessary since, on the one hand, no reliable chemical reaction data is available for such a set up of coupled ideal reactors and, on the other hand, the model is not able to cope with local conditions due to low resolution and the lack of adequate models. For example, the oxidation of mixed gaseous hydrocarbons (i.e. in this case $\mathrm{CH}_{4}$ only) is mass transfer limited and therefore, an ideal mixed reactor needs to be adapted using the averaged chemical reaction kinetics.

The rate of degradation (massflow) for each component is calculated using the relationship given with equation (15). 


$$
\dot{m}_{j}=k V \lambda_{j} M_{j} \sum_{l}^{k=1} C_{k}^{\lambda_{k}}
$$

In this relationship, the velocity of reaction $k$ is coupled with the volume of the reaction element $V$, the molar mass $M$ and the stoichiometric cofficient $\lambda$ for each component (index $\mathrm{j}$ over all components). The approach is reactant limited assuming an elementary reaction order. Hence, the molar density $C$ to the power of the stoichiometric coefficient $\lambda$ sums up over all reactants $\mathrm{k}$ of a reaction.

For the bed phase, the degradation of $\mathrm{CaCO}_{3}$ is implemented. The chemical reaction is given with equation (16).

$$
\mathrm{CaCO}_{3} \Longrightarrow \mathrm{CaO}+\mathrm{CO}_{2}
$$

Energy is chemically provided by the combustion of $\mathrm{CH}_{4}$, equation (17) holds.

$$
\mathrm{CH}_{4}+2 \mathrm{O}_{2} \Longrightarrow \mathrm{CO}_{2}+2 \mathrm{H}_{2} \mathrm{O}
$$

In principle, various reactions are possible, as long as the defined medium covers all components and the stoichiometric coefficients are defined.

\subsection{Mechanisms of heat transfer}

For the heat transfer between the gas and bed phase as well as the isolation shell convectional and radiational mechanisms are implemented. For convectional heat transfer between the gas and its corresponding exchange partner, the heat transfer coefficient $\alpha$ is dependent on the Reynolds number Re, the Prandtl number $P r$ and the Nusselt number $N u$. The general convectional heat transfer equation in terms of $\alpha$ is shown with equation (18).

$$
\dot{Q}_{\text {conv } 1 \leftrightarrow 2}=\alpha_{12} A_{12}\left(T_{1}-T_{2}\right)
$$

Various heat transfer models for the gas phase solid interaction are implemented in the Modelica fluid library [1]. Additionally, two relationships for Nusselt numbers are given in [10]. The equations are shown with (19) and (20).

$$
\begin{aligned}
N u= & \frac{\xi / 8(\operatorname{Re}-1000) \operatorname{Pr}}{1+12.7 \sqrt{\xi / 8}\left(\operatorname{Pr}^{2 / 3}\right)}\left[1+\left(\frac{D_{h}}{L}\right)^{\frac{2}{3}}\right] \\
\xi= & \left(1.82 \cdot \log _{10}(\operatorname{Re})-1.64\right)^{-2} \\
& 2300<\operatorname{Re}<10^{6}, D_{h} / L<1
\end{aligned}
$$

Within small deviations in the range of $P r$, $0.5<\operatorname{Pr}<1.5$, the simplified equation (21) can be used.

$$
N u=0.0214\left(\operatorname{Re}^{0.8}-100\right) \operatorname{Pr}^{0.4}\left[1+\left(\frac{D_{h}}{L}\right)^{\frac{2}{3}}\right]
$$

All the models calculate $\alpha$ in a similar range from $8-10 \mathrm{~W} / \mathrm{m}^{2} \mathrm{~K}$.

Convectional heat transfer between the bed phase and the isolation is also realized with the heat transfer coefficient $\alpha$ and equation (18). $\alpha$ is calculated from various process parameters using two different approaches which are described in detail in [8]. Typical values are said to be $50 \leq \alpha \leq 200 \mathrm{~W} / \mathrm{m}^{2} \mathrm{~K}$

Also, models with constant $\alpha$ are possible to choose. The heat transfer models are designed to be replaceable in order to enable the choice between different heat transfer mechanisms as described above.

Radiation between the bed, the gas and the isolation is modeled using equation (22).

$$
\dot{Q}_{\text {rad } 1 \leftrightarrow 2}=\varepsilon_{12} A_{12} \sigma\left(T_{1}^{4}-T_{2}^{4}\right)
$$

The referenced area for radiational heat transfer between transfer object 1 and 2 is given with $A_{12}$. The emissivity coefficient $\varepsilon_{12}$ relates the visibilty between area $A_{12}$ and $A_{21}$ and the emissivity of corresponding objects. There are three emissivity coefficients to be calculated, namely wall $\rightarrow$ bed (wb), wall $\rightarrow$ gas (wg) and bed $\rightarrow$ gas (bg). The formulas for calculating these coefficients are given with equation (23) to (26).

$$
\begin{aligned}
\varepsilon_{w b}= & \frac{\varepsilon_{w} \varepsilon_{b}\left(1-\varepsilon_{g}\right)}{U} \\
\varepsilon_{w g}= & \frac{\varepsilon_{w} \varepsilon_{g}\left(1+\Phi\left(1-\varepsilon_{g}\right)\left(1-\varepsilon_{b}\right)\right)}{U} \\
\varepsilon_{b g}= & \frac{\varepsilon_{b} \varepsilon_{g}\left(1+\Phi\left(1-\varepsilon_{g}\right)\left(1-\varepsilon_{w}\right)\right)}{U} \\
U= & 1-\left(1-\varepsilon_{g}\right)\left(1-\varepsilon_{w}\right) \\
& \left(1-\Phi\left(1-\left(1-\varepsilon_{b}\right)\left(1-\varepsilon_{g}\right)\right)\right)
\end{aligned}
$$

The variable $\Phi$ is defined as the ratio between the free bed area (surface) and the free isolation area. Details about the derivation can be found in [4]. The values of emissivity are almost constant over length $x$ and temperature $T$ with $\varepsilon_{w b}=0.184, \varepsilon_{w g}=0.695$ and $\varepsilon_{b g}=0.615$. 


\section{Computational results}

The model is designed and numerically integrated within the Dymola modeling environment. The set up is depicted in the Figure 3.

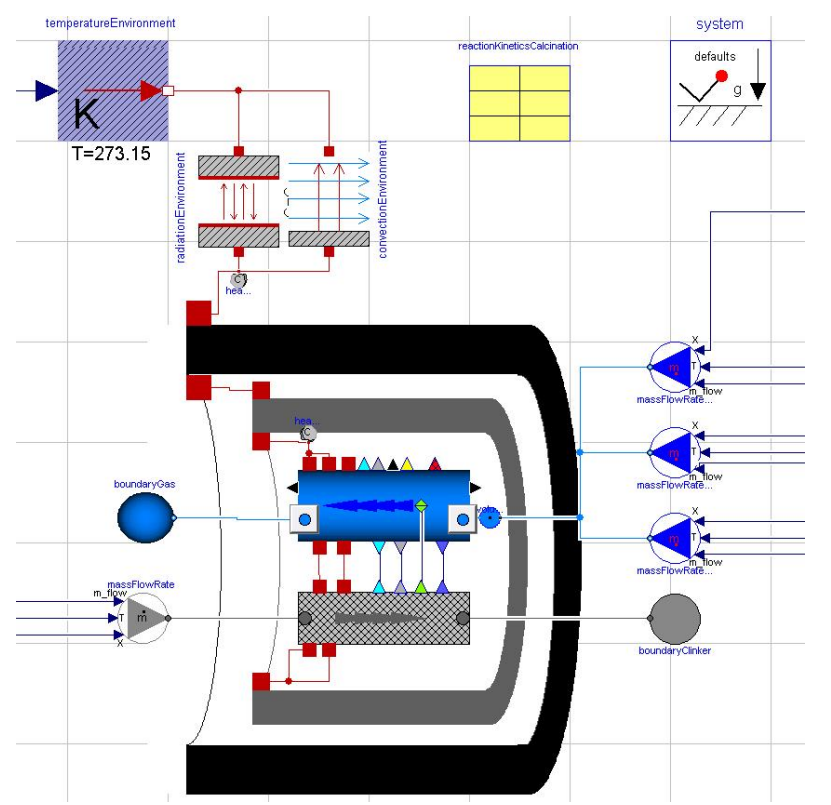

Figure 3: Dymola model of a rotary kiln

The black and the grey objects denote the steel and isolation shell, respectively, with different geometric and material parameters. Inside the pipe, there is counter current flow of the gas phase (blue element) and the bed phase (limestone material, grey element). Different mechansims for heat and mass transfer as well as chemical reactions are chosen as described earlier in this paper. Further components, e.g. crust of semi liquefied limestone, can be added but are not modeled in this contribution.

The calcination process (degradation of $\mathrm{CaCO}_{3}$ ) is shown in Figure 4.

The system is initialized at $273.15 \mathrm{~K}$ and atmospheric pressure (time [s]: 0-3000). In a next step, the system is brought to the operating point by increasing temperature of the incoming mass flows of the bed and the gas phase (time [s]: 3000-13000). This procedure is followed by the intial increase of the combustion gas massflow (time [s]: 13000-250000, massflow step $[\mathrm{kg} / \mathrm{s}]: 0.1-1.2692)$. It is clear to see that the rate of $\mathrm{CaCO}_{3}$ degradation intensifies as the temperature increases. A second step to the combustion gas massflow is applied after 300000 seconds (massflow step [kg/s]: 1.2692-2.5392). Again, the degradation of $\mathrm{CaCO}_{3}$ increases. The increase of degradation, while applying these steps to the combustion gas flow, is due to the

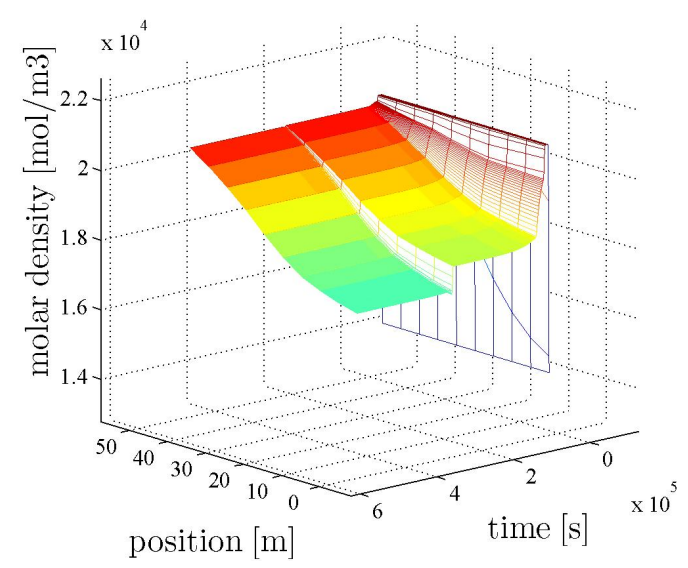

Figure 4: Degradation of $\mathrm{CaCO}_{3}$ through calcination

increase of temperature and the endothermic character of the calcination reaction. The increase of temperature for the main components of the rotary kiln (steel, isolation, gas and bed phase) is depicted in Figure 5

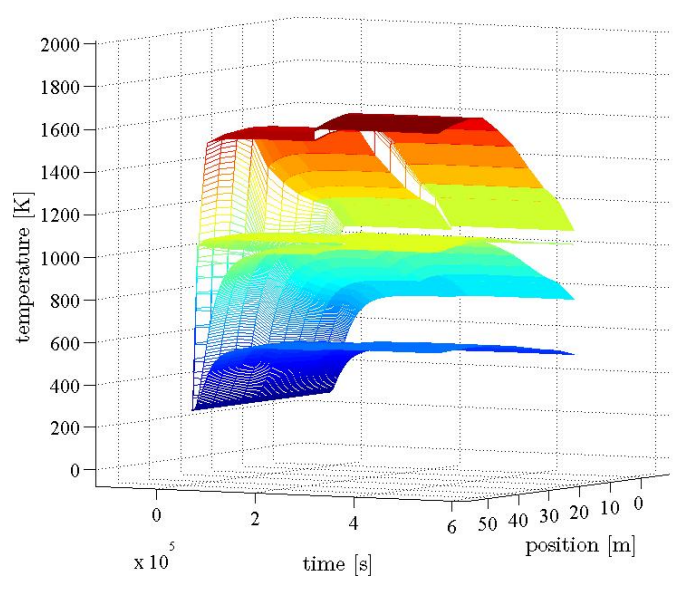

Figure 5: Temperature of steel, isolation, bed and gas phase

While the gas phase (red) responds extremely fast to the step in combustion gas massflow, the bed phase (yellow) is slower. The isolation (turquoise) and steel (blue) shell are even slower in their step response. The different time constants of the process can be recognized by an exemplary inspection of Figure 6 to 7 .

It is easy to observe the small time constant of the gas response lying in the range of 10-200 seconds.

For the isolation shell, the time constant of reponse lies in the range of 20000-30000 seconds. This explains the stiffness of the system and the necessity of stiff numerical solvers for simulations. Furthermore, these time constants correspond to the known time constants from process industry, which implies that 


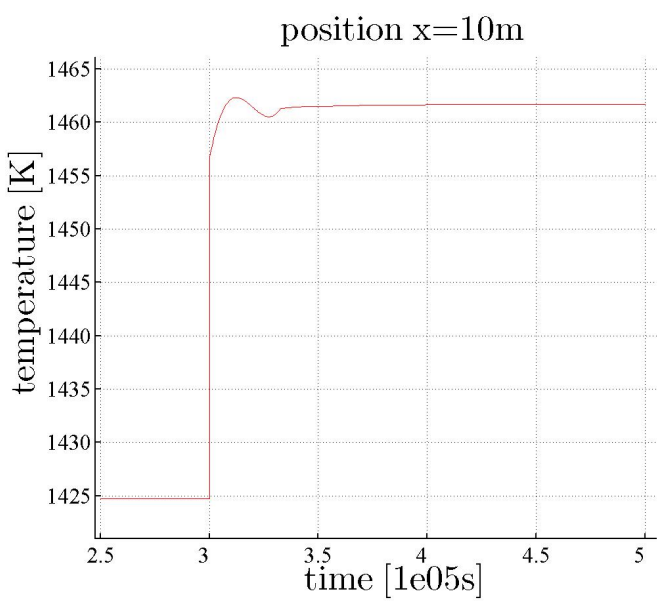

Figure 6: Temperature response of gas phase due to combustion gas step

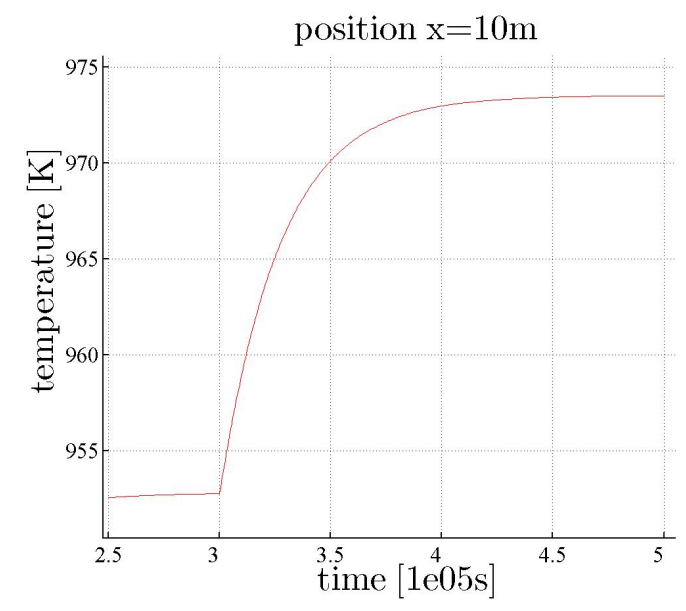

Figure 7: Temperature response of isolation due to combustion gas step

the dynamical behaviour of the model reliably represents the calcination process. Although the steady state values of the operating point also correspond to the known values from process industry, this modeling approach is justified even more by comparing its values to CFD simulation results in the following chapter.

\section{Comparison to CFD simulations}

Computational fluid dynamics (CFD) is a widely accepted tool for the detailed description of gaseous combustion phenomena occurring within a rotary kiln [6],[3]. Unfortunately, it does neither allow for the modeling of transport of the solid bed nor for the theoretical description of the chemical reactions therein. In the current work, different sub-models have been integrated in the commercial CFD code Fluent in order to model the dynamics of the granular flow of the lime- stone particles and the coupling of gas and solid phase. Since this work mainly focuses on the 1-D model of the limestone calcination process, only a short summary of the models used in the CFD simulations will be given here.

\subsection{Introduction of the CFD rotary kiln model}

The three dimensional computational domain of the freeboard in the kiln is bounded by the refractory and the surface of the solid bed. The methane burner extends into the kiln on one end of the drum (Figure 8).

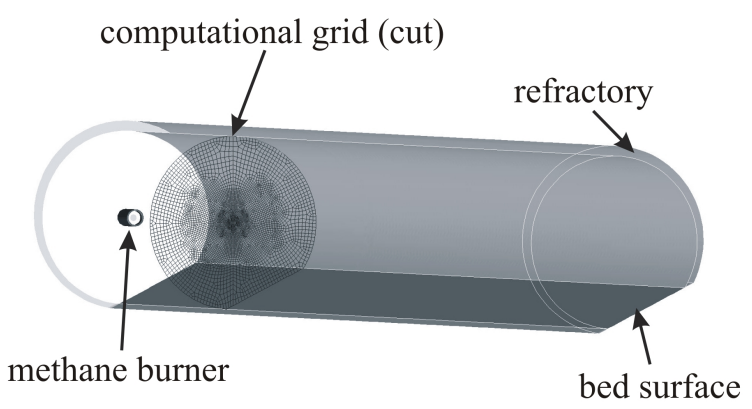

Figure 8: 3-D computational domain of the limestone rotary kiln

Steady state conservation equations of the compressible gas flow are solved considering the realizable $\mathrm{k}-\varepsilon$-model for turbulent effects in the gas phase. Transport of four separate species $\left(\mathrm{O}_{2}, \mathrm{CH}_{4}, \mathrm{CO}_{2}\right.$ and $\mathrm{H}_{2} \mathrm{O}$ ) is calculated explicitly in the gas phase, while the fifth one $\left(\mathrm{N}_{2}\right)$ sums to unity. Combustion rates of methane oxidization are assumed to be limited by the mixing of turbulent eddies and thus, the eddydissipation model is used to predict reaction rates of the volumetric reactions in the gas phase. Energy transport phenomena include conduction, convection and radiation ( $\mathrm{P}-1$ model), while the latter plays the major role in rotary kiln processes. Absorption of the gas mixture is mainly affected by the product constituents of the gas $\left(\mathrm{CO}_{2}\right.$ and $\left.\mathrm{H}_{2} \mathrm{O}\right)$. Therefore the wsggm-cell-based model is applied to calculate the local absorption coefficient of the gas mixture. A significant amount of energy discharges into the environment. Conduction driven heat transfer through the refractory is being calculated explicitly by applying a computational grid to this region also. On the outer 
surface of the kiln two heat transfer mechanisms are considered: convection and radiation.

Thermal coupling between gas and solid phase is realized by setting a temperature profile on the bed surface. For this purpose mass, species and energy conservation equations within the particulate solid bed are solved by implementing a mathematical submodel for the solid bed. Since it has recently been proven, that axial mixing can be neglected in industrial rotary kiln processes, transport of the solid bed can be simplified by assuming a plug flow [9]. Additionally the bed is assumed to be well mixed locally in any given cross section.

Limestone calcination can be modeled as a shrinking core process with surface reaction control according to equation (27) [7].

$$
\frac{d m_{\mathrm{CaCO}_{3}}}{d t}=-k_{0} \exp \left(-\frac{E_{\mathrm{A}}}{R T_{p}}\right) \cdot 4 \pi r_{p}^{2} N_{p} M_{\mathrm{CaCO}_{3}}
$$

Herein $r_{p}$ and $N_{p}$ represent the mean particle radius and the total number of limestone particles, respectively. $\mathrm{CO}_{2}$ released by the calcination reaction is assumed to be transported instantaneously to the hot flow by neglecting any transport resistance in the solid bed. It is released in the computational space of the hot gas flow by adding mass and species sources in the cells adjacent to the bed surface.

In Figure 9 the contour plot of the local gas temperature on the center plane is shown as an exemplary result of the CFD simulations.

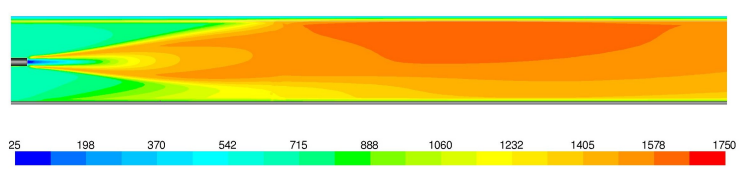

Figure 9: Contour plot of the local gas temperature on the center plane in $[\mathrm{K}]$

In order to justify the 1-D abstraction of the process modeled within the Dymola environment, the computational results are compared to the highly resolute results of the CFD simulations. For this purpose, volume based average values $\bar{\Theta}_{V, j}$ of the CFD simulation data are calculated for a discrete number of slices in the gas phase. Due to the strongly anisotropic flow field in the vicinity of the burner mass and velocity weighted average has to be applied for the potential variables. This is shown in equation (28).

$$
\bar{\Theta}_{V, j}=\frac{\sum_{N_{\text {cells }}} \Theta_{i} \rho_{i} A_{i} v_{i, a x}}{\sum_{N_{\text {cells }}} \rho_{i} A_{i} v_{i, a x}}
$$

In equation (28), $V_{i}$ as the volume of each single cell inside the discrete slice and $v_{i, a x}$ as the local axial velocity of the gas mixture are used to calculate the volume based average of slice $j$ for each scalar $\Theta$.

Since plug flow has been assumed in the clinker bed, equation (28) can be simplified to the volume weighted average for all potential variables in the solid phase as given in equation (29).

$$
\bar{\Theta}_{V, j}^{s}=\frac{\sum_{N_{\text {cells }}} \Theta_{i} V_{i}}{\sum_{N_{\text {cells }}} V_{i}}
$$

In contrast, flow variables such as reaction rates need to be integrated within each single control volume in the gas and in the solid domain, respectively. For these data equation (30) is valid.

$$
\Theta_{V, j}=\sum_{N_{\text {cells }}} \Theta_{i}
$$

\subsection{Comparison}

Geometrical parameters and boundary conditions are chosen to be equal in both simulation environments. In addition, the free chemical reaction parameters $\left(B, n, E_{a}\right)$ for adapting the model are fitted to CFD simulation data by applying a least square fit using equation (15). Therefore, the CFD simulation data are concentrated in larger 1-D cells by applying the above described weighting functions. After model adaption via least square fit, a Dymola simulation to steady state is performed. The simulated data from the Dymola simulation environment are then compared to the weighted, concentrated CFD simulation data. The essential values of the process are compared. This includes temperature profile of gas and bed phase, molar densities of $\mathrm{O}_{2}$ and $\mathrm{CH}_{4}$ in the gas phase as well as molar density of $\mathrm{CaCO}_{3}$ in the bed phase (controlled variable for future model predictive control).

Figure 10 shows the temperature profiles of the gas phase for Dymola and CFD simulation.

In the next figure (Figure 11), the consumption of $\mathrm{O}_{2}$ and $\mathrm{CH}_{4}$ is depicted.

The figures show a similar behaviour of the gas phase in both simulation environments. For the error $e$ holds $e<2 \%$. On first inspection, this seems to be a good result. Nevertheless, the more relevant data for intended controlling of the rotary kiln process is the temperature and the concentration of components in 


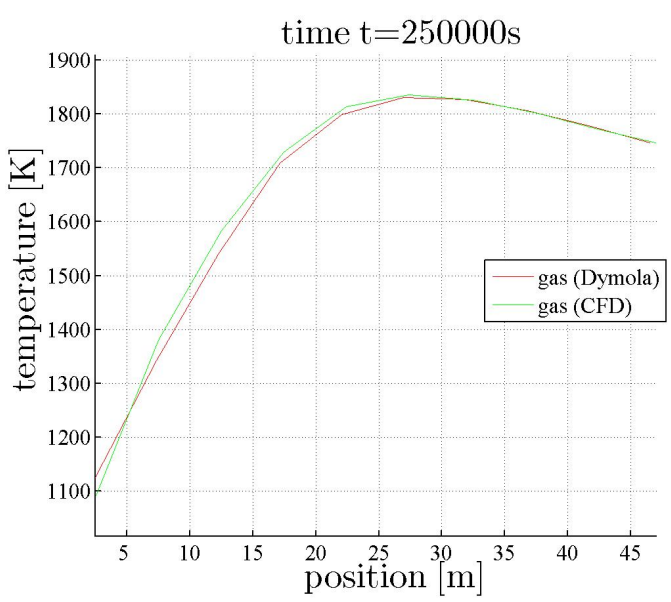

Figure 10: Comparison of gas phase temperature

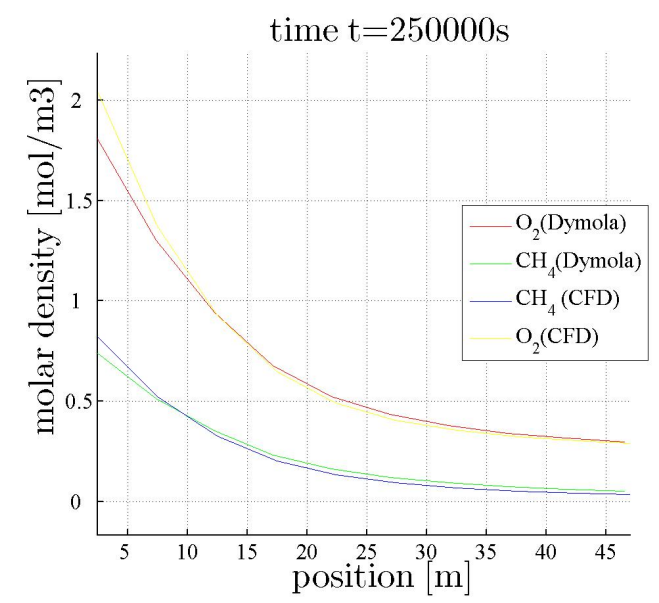

Figure 11: Comparison of gas phase molar density of $\mathrm{O}_{2}$ and $\mathrm{CH}_{4}$

the bed phase. Figure 12 shows the temperature of the bed phase.

Despite the different scaling of the graph, for the error $e$ holds $e<3 \%$. The next figure (Figure 13) shows the degradation of $\mathrm{CaCO}_{3}$ in the bed phase using the molar density as depicted variable. The maximum error for the molar density of $\mathrm{CaCO}_{3}$ is $e=6.6 \%$. Although this value is slightly higher than the previous errors, the 1-D modeling approach is successfully applied. Various alternative values of the process were compared, yielding to the same outcome. The main advantage of the 1-D modeling approach is the possiblity of faster simulations while deviations are kept small enough in the scope of robustness in terms of observer based model predictive control. While the simulation until convergence in the CFD environment takes up to several days, the simulation of the Dymola model takes seconds to minutes depending on the change of input variables. Another enormous advantage of the 1-

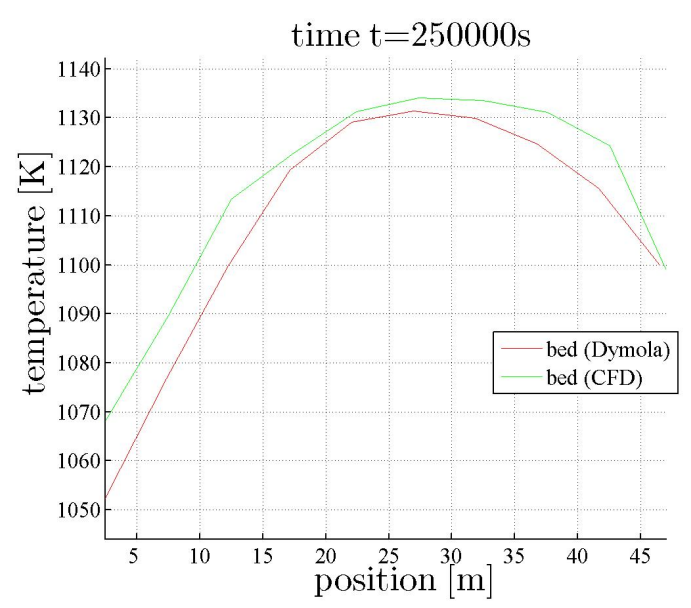

Figure 12: Comparison of bed phase temperature

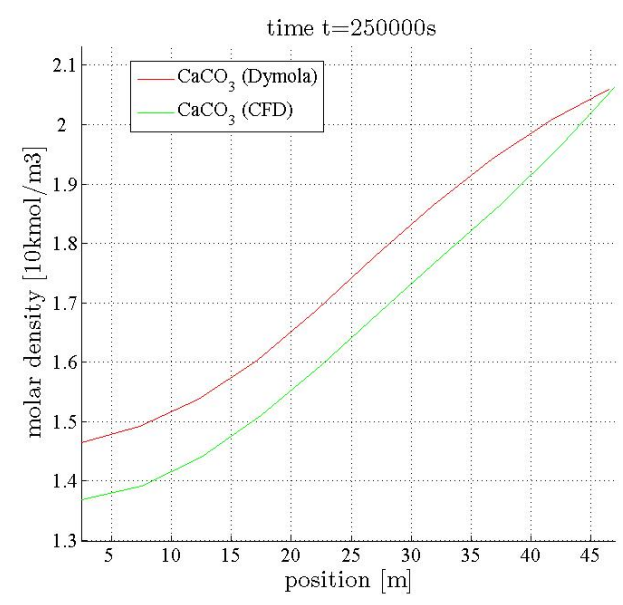

Figure 13: Comparison of bed phase molar density of $\mathrm{CaCO}_{3}$

D modeling approach is the use of the $1^{\text {st }}$ principles for physical abstraction. Since the identified data based models are only valid in the range of measured data, the nonlinear 1-D model of the rotary kiln will have a wider range of validation. Furthermore, the simulated data sets are always open to physical interpretation and hence, are easier to check for plausibility.

\section{Outlook}

In a first step, the complex counter current flow process of rotary kilns is modeled and applied to the limestone degradation. First results show reasonable behaviour of the process physics. The comparison to CFD simulation data confirms these results. The essential process parameters are compared and the errors are small enough to allow for application in observer based model predictive control application. In addi- 
tion, the modeling approach has a lower computational burden and results in linearizations which are capable of real-time application in an expert system.

For the future, details in both models will be refined in order to get even better results. Furthermore, the model is going to be enhanced with a particle burner and applied to the chemically more complex cement production process. The task of automatic least square fit for the free chemical parameters of the model will be enhanced in order to cover the more complex cement production process. A detailed report on both topics will soon be published. Alongside with the enhanced model, linearizations of the nonlinear model in desired operating points will be derived. Using these linearizations, an observer for immeasurable process values will be established. Furthermore, a model predictive control will be designed in order to hold a desired operating point of the plant.

\section{References}

[1] F. Casella, H. Tummescheit, and M. Otter. Modelica fluid library www.modelica.org/libraries/ modelica_fluid/releases/1.0, 2009.

[2] H. Elmqvist, H. Tummescheit, and M. Otter. Object-oriented modeling of thermo-fluid systems. Modelica Association, November 2003.

[3] M. Georgallis, P. Nowak, M. Salcudean, and I.S. Gartshore. Modelling the rotary lime kiln. Canadian Journal of Chemical Engineering, 83(2):212-223, 2005.

[4] R. Jeschar, R. Alt, and E. Specht. Grundlagen der Wärmeübertragung. Viola-Jescher Verlag, 1990.

[5] H. Kramers and P. Croockewit. The passage of granular solids through inclined rotary kilns. Chemical Engineering Science, 1(6):259-265, 1952.

[6] F. Marias, H. Roustan, and A. Pichat. Modelling of a rotary kiln for the pyrolysis of aluminium waste. Chemical Engineering Science, 60:46094622, 2005.

[7] K.S. Mujumdar, K.V. Ganesh, S.B. Kulkarni, and V.V. Ranade. Rotary cement kiln simulator (rocks): Integrated modeling of pre-heater, calciner, kiln and clinker cooler. Chemical Engineering Science, 62:2590-2607, 2007.
[8] A. Queck. Untersuchung des gas- und wandseitigen Wärmetransportes in die Schüttung von Drehrohröfen. PhD thesis, Otto-von-GuerickeUniversität Magdeburg, 2002.

[9] R.G. Sheritt, J. Chaouki, A. Mehrotra, and L. Behie. Axial dispersion in the three-dimensional mixing of particles in a rotating drum reactor. Chemical Engineering Science, 58(2):401-415, 2003.

[10] VDI-Gesellschaft Verfahrenstechnik und Chemieingeniuerwesen, editor. VDIWärmeatlas. Springer, 8th edition, 1997.

\section{Acknowledgement}

The authors gratefully thank the Federal Ministry of Education and Research for funding BMBF 3257 MoProOpt. Furthermore, we highly appreciate the supervision by the Project Management Agency Forschungszentrum Karlsruhe (PTKA). 